\title{
Erratum to: Tubulin-related cerebellar dysplasia: definition of a distinct pattern of cerebellar malformation
}

\author{
Romina Romaniello $^{1}$ - Filippo Arrigoni ${ }^{2} \cdot$ Elena Panzeri $^{3}$ - Andrea Poretti ${ }^{4,5}$. \\ Alessia Micalizzi ${ }^{6,7}$ - Andrea Citterio ${ }^{3}$ - Maria Francesca Bedeschi ${ }^{8}$ - Angela Berardinelli ${ }^{9}$. \\ Raffaella Cusmai ${ }^{10}$ - Stefano D'Arrigo ${ }^{11}$ - Alessandro Ferraris ${ }^{12}$ • Annette Hackenberg ${ }^{13}$. \\ Alma Kuechler ${ }^{14}$ • Margherita Mancardi ${ }^{15}$ - Sara Nuovo ${ }^{6,16}$ • Barbara Oehl-Jaschkowitz ${ }^{17}$. \\ Andrea Rossi ${ }^{18}$ - Sabrina Signorini ${ }^{9}$ - Frank Tüttelmann ${ }^{19}$ • Dagmar Wahl ${ }^{20}$ - Ute Hehr ${ }^{21}$. \\ Eugen Boltshauser ${ }^{22} \cdot$ Maria Teresa Bassi $^{3} \cdot$ Enza Maria Valente $^{6,23} \cdot$ Renato Borgatti $^{1}$
}

Published online: 12 September 2017

(C) European Society of Radiology 2017

\section{Erratum to: Eur Radiol}

https://doi.org/10.1007/s00330-017-4945-2
The original version of this article, published on 4 July 2017 , unfortunately contained a mistake. The following correction has therefore been made in the original:

The first name of the author Raffaella Cusmai was rendered incorrectly and has now been corrected.

The online version of the original article can be found at http://doi.org/10. 1007/s00330-017-4945-2

Enza Maria Valente enzamaria.valente@unipv.it

Renato Borgatti renato.borgatti@bp.lnf.it

1 Neuropsychiatry and Neurorehabilitation Unit, Scientific Institute, IRCCS Eugenio Medea, Via D. L. Monza 20, 23842 Bosisio Parini, Lecco, Italy

2 Neuroimaging Laboratory, Scientific Institute, IRCCS Eugenio Medea, Bosisio Parini, Lecco, Italy

3 Laboratory of Molecular Biology, Scientific Institute, IRCCS Eugenio Medea, Bosisio Parini, Lecco, Italy

4 Section of Pediatric Neuroradiology, Division of Pediatric Radiology, Russell H. Morgan Department of Radiology and Radiological Science, The Johns Hopkins University School of Medicine, Baltimore, MD, USA

5 Department of Neurogenetics, Kennedy Krieger Institute, Baltimore, MD, USA

6 Neurogenetics Unit, IRCCS Fondazione Santa Lucia, Rome, Italy

7 Department of Biological and Environmental Sciences, University of Messina, Messina, Italy

8 Clinical Genetics Unit, Fondazione IRCCS Ca' Granda, Ospedale Maggiore Policlinico, Milan, Italy

9 Unit of Child Neurology and Psychiatry, IRCCS "C. Mondino" Foundation, Pavia, Italy
10 Neurology Unit, Bambino Gesù Children's Hospital, IRCCS, Rome, Italy

11 Developmental Neurology Division, IRCCS Fondazione Istituto, Neurologico C. Besta, Milan, Italy

12 Mendel Laboratory, IRCCS Casa Sollievo della Sofferenza, San Giovanni Rotondo, Italy

13 Universitäts, Kinderspital Zürich, Eleonorenstiftung, Zürich, Switzerland Essen, Germany

15 Unit of Child Neuropsychiatry Giannina Gaslini Institute, Genoa, Italy

16 Department of Medicine and Surgery, University of Salerno, Salerno, Italy

17 Practice of Human Genetics, Homburg (Saar), Germany

18 Neuroradiology Unit, Giannina Gaslini Institute, Genoa, Italy

19 Institute of Human Genetics, University of Münster, Münster, Germany

20 Private Practice for Human Genetics, Augsburg, Germany

21 Department of Human Genetics, University of Regensburg, Regensburg, Germany

22 Department of Pediatric Neurology, University Children's Hospital, Zurich, Switzerland

23 Department of Molecular Medicine, University of Pavia, Pavia, Italy
14 Institut für Humangenetik Essen, Universität Duisburg-Essen, 